\title{
Clinical evaluation of the loop-design fibre-reinforced composite and the band-and-loop space maintainers.
}

\begin{abstract}
Purpose: The fibre-reinforced composite space maintainer (FRCSM) has been suggested as an alternative to the band-and-loop space maintainer (BLSM). The aim of this in-vivo study was to evaluate the clinical performance and the reasons for failure of the two types of fixed space maintainers over a six-month period.
\end{abstract}

Methods: Twenty patients, ranging from 4-9 years old, were selected for this study. They were randomly divided into two groups $(n=10)$ according to the type of space maintainer that was placed. The patients were recalled on a monthly basis for clinical evaluation over a period of six months. The two-sample t-test and the non-parametric Wilcoxon rank sum test were used for statistical analysis.

Results: Both groups of space maintainers had a $50 \%$ failure rate. The main reason for BLSM failure was bending of the wire with impingement on the soft tissue. The FRCSM failed due to debonding and fracture of the fibre loop. There was no statistically significant difference between the failure rates $(P=0.53)$.

Conclusion: The clinical performances of both space maintainers were disappointing. Only $50 \%$ of fixed space maintainers were still clinically acceptable according to the strict evaluation criteria used. Further research is recommended on the loop-design FRCSM.

Key words: Space maintenance, Band-and-loop space maintainer, Fibre-reinforced composite space maintainer

1. Nicoline Potgieter: $B C h D(U P), P G$ Dip Dent: Endodontics(UP), PG Dip Paedodontics(UWC), MSc Dent(UP). Head of Division Paediatric Dentistry, Department of Odontology, University of Pretoria.

2. Paul D Brandt: BChD (UP), MSc Odont (UP), Adv Dip Aesthetic Med. Head of Division Dental Materials. Department of Odontology, University of Pretoria.

3. Nadia Mohamed: BChD(Stell), BScHons(Stell), MSc in Dental Sciences(Stell), PhD(Stell). Head of Department Paediatric Dentistry,University of the Western Cape.

\section{Corresponding author}

Nicoline Potgieter:

Department of Odontology, School of Dentistry, Faculty of Health

Sciences, PO Box 1266, Pretoria 0001, South-Africa. Tel: 0123192932

Fax: 012326 2754. Cell: 082921 9645. E-mail: nicoline.potgieter@up.ac.za

\section{ACRONYMS}

BLSM: band-and-loop space maintainer

FRCSM: fibre-reinforced composite space maintainer

\section{INTRODUCTION}

Loss of space due to drifting after early loss of deciduous teeth is one of the leading causes of malocclusion in paediatric patients in the deciduous- or transitionaldentition stages. ${ }^{1}$ An effective space-maintaining appliance could, therefore, reduce the incidence of occlusal discrepancies. ${ }^{2}$

Stainless steel band-and-loop maintainers (BLSMs) are widely used as fixed appliances to maintain space after the early loss of a single deciduous tooth and should remain in place until the permanent tooth erupts. ${ }^{3}$ Common reasons for the failure of BLSMs are fracturing and bending of the loop or loosening of the band under occlusal forces. ${ }^{4,5}$ The average "survival time" of a BLSM has been reported as approximately 13 months. ${ }^{6}$ A long-term study by Sasa et al. ${ }^{7}$ revealed the success rates of BLSMs to approximate only 10 percent. Thus, alternatives to the BLSM are being investigated.

Fibre-reinforced composite material is known for its flexural and physical strength. ${ }^{3,8}$ Consequently, the fibrereinforced composite space maintainer (FRCSM) has been suggested as an alternative to the conventional stainless steel BLSM. ${ }^{1,3,9,10}$

A mean survival time of five months for FRCSMs has been reported ${ }^{4,11}$ and success rates over a six-month period were found to range from $27.5 \% 4$ to $67 \% .^{1}$ These are relatively low survival times and further research on the FRCSM placement technique has been recommended. ${ }^{4}$ Kulkarni et al. ${ }^{3}$ and Yeluri et al..$^{9}$ tested the loop-design FRCSM in vitro and concluded that it might be considered an alternative to BLSMs.

This study presents a novel placement technique of the loop-design FRCSM. The study aimed to investigate and compare the in vivo failure rates, as well as the reasons for failure, of the loop-design FRCSM and the metal BLSM over a six-month period. 


\section{MATERIALS AND METHODS}

Twenty patients, ranging from 4-9 years old, were selected for this study, determined by strict criteria. Patients were included provided they presented with: premature loss of a deciduous first molar (>one year before the expected exfoliation time); anchor teeth (second deciduous molars) with intact, undamaged buccal and lingual surfaces to bond to; and anchor teeth with more than half of the root length present. ${ }^{1,4,10}$ Patients were excluded from the study if they presented with: teeth with compromised structure in the intended bonding area (i.e. demineralized enamel, caries, fractures, iatrogenic damage or existing restorations); occusal discrepancies (i.e. a cross-bite, an open bite, or a deep bite) $)^{1,4,10}$ or the inability to return for monthly follow-up appointments.

The space maintainers were allocated alternately to patients in the order in which they were accepted for the study. The sample was therefore randomly divided into two groups ( $n=10)$ according to the type of space maintainer that was placed. The patients were recalled on a monthly basis for clinical evaluation for six months.

\section{ETHICAL CONSIDERATIONS}

The study was approved by the Research Committee of the School of Dentistry at the University of Pretoria and the Ethics Committee of the University (ref. nr 523/2015). Written informed consent was obtained from the parent/legal guardian of each child who participated. Participants over the age of seven years also gave their own informed assent.

\section{CLINICAL PROCEDURES}

The principal investigator personally selected all participants, placed all space maintainers, and performed all follow-up procedures. The placement techniques used are detailed below.

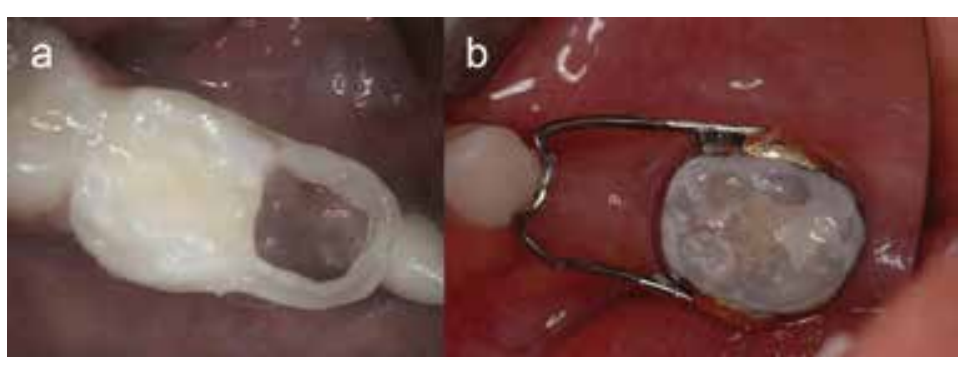

Figure 1: Examples of (a) a FRCSM; (b) a BLSM immediately after placement.

\section{PLACEMENT OF THE FRCSMS}

The FRCSMs in this study differed from the design of the FRCSMs tested in previous clinical studies.,2,10,11 To eliminate any restriction of normal physiological tooth movements and growth of the jaws, the teeth adjacent to the edentulous area were purposefully not bonded together when the loop was constructed.

The FRCSMs were placed according to a step-wise clinical procedure. The anchor tooth (second deciduous molar), the edentulous area and the tooth anterior to the edentulous area (deciduous canine) were isolated with rubber dam. A matrix band was applied to the canine and the anchor tooth was prepared by cleaning the surfaces intended for bonding using pumice, water and a rubber polishing cup to remove all plaque and surface accumulations.

As recommended by Zilberman, ${ }^{12}$ the bonding surfaces on the second deciduous molars were prepared by etching the enamel for $60 \mathrm{sec}$ with 34 percent phosphoric acid (Scotchbond Universal etchant, 3M ESPE, St. Paul, USA). The everStick instruction manual recommends a $60 \mathrm{sec}$ etch time to maximize bond strength..$^{13}$ Bonding agent (Adper Scotchbond 1XT adhesive, 3M ESPE, St. Paul, USA) was applied and light cured according to the manufacturer's instructions.

The uni-directional glass-fibre bundle (everStick C\&B, Stick Tech Ltd., Turku, Finland) was placed in a continuous loop extending from the buccal to the lingual surfaces of the anchor tooth. The full buccal and lingual dimensions of the anchor tooth were used, the bundle being placed in the middle of the occluso-gingival dimension. The glass-fibre bundle was secured in position with a flowable composite (Filtek Supreme XTE Flowable, 3M ESPE, St. Paul, USA) and light cured on both the buccal and lingual surfaces for an initial $10 \mathrm{sec}$ period. The loop was then manipulated and shaped to the ideal form.

The glass fibre was then moistened with unfilled adhesive resin ${ }^{9}$ (Adper Scotchbond $1 \mathrm{XT}$ adhesive, 3M ESPE, St Paul, USA), using a bond applicator brush and the entire loop was cured for 40 sec. Flowable composite (3M ESPE) was applied to cover the whole loop and light-cured for $40 \mathrm{sec}$. An ELiTEDENT ${ }^{\circledR}$ Q-4 LED curing light was used, and the curing tip was kept within one $\mathrm{mm}$ of the material to ensure a complete cure. The curing light was regularly tested with a Bluephase ${ }^{\circledR}$ meter to ensure a consistent output of 1000-1100 mW/cm². The FRCSMs were finished and polished using a yellow striped, flame-shaped diamond finishing bur (Dentsply Sirona, Switzerland; ISO 806314249504 012) and the Enhance polishing system (Dentsply Sirona, Milford, USA) (Figure 1a).

\section{PLACEMENT OF THE BLSMS}

The BLSMs were placed according to the standard clinical procedure. An orthodontic band was fitted around the anchor tooth and an impression was taken with the band in place. The band was then transferred to the impression and secured before the impression was sent to the laboratory for pouring of the model and manufacturing of the space maintainer. The BLSMs were cemented with glass ionomer cement (GIC) (Fuji ORTHO, GC America, Illinois, America) according to the manufacturer's instructions (Figure 1b).

\section{Follow-up and evaluation of space maintainers} Monthly follow-up appointments were scheduled over a sixmonth period. Parents and patients were, however, instructed to report immediately for an emergency appointment if any problem or failure occurred between these pre-arranged appointments. This ensured that the timing and reasons for any failure of appliances were accurately recorded.

\section{Failure criteria for a space maintainer}

Based on previous clinical comparative studies, ${ }^{1,10}$ a space maintainer was classified as having failed when it presented with any of the following:

- Debonding of the fibre-composite or the band-cement interface; 
- Debonding of the enamel-composite or the cementenamel interface;

- Fracture of the fibre/metal frame; or

- Bending of the fibre/metal loop to the extent that the device was in contact with the soft tissue.

\section{STATISTICAL ANALYSIS}

Frequency tables were used to report on the monthly failures and repairs per group. Contingency tables were drawn up to highlight the distributions of the space maintainers according to the age of the appliance and the reasons for failure. Statistical differences between failure rates of groups were compared and analysed using mean (two sample t-test) and median (non-parametric Wilcoxon rank sum test) values.

\section{RESULTS}

Failures and reparability of failed appliances During the six-monthly follow-up period, five out of ten (50 percent) of both types of space maintainers failed according to the strict failure criteria.

\section{A comparison of failure rates between the FRCSMs and BLSMs}

Table 1 provides a summary of the statistical analysis comparing failure rates of the two groups. The number of days until failure was used as an indication of longevity of the appliance. All space maintainers that survived the six-month period therefore survived 180 days. The difference between the two mean values was 5.1, which was not statistically significant $(P=0.525)$. The difference between the median values was 16 , also not statistically significant $(P=0.620)$.

\begin{tabular}{|c|c|c|c|}
\hline & FRCSM & BLSM & p-values \\
\hline$n$ & 10 & 10 & \\
\hline Mean (+-SD) & $145.6(46.53)$ & 150.7 (48.94) & $0.525^{\star}$ \\
\hline Median (IQR) & $164(136-180)$ & $180(146-180)$ & $0.620^{\star \star}$ \\
\hline Min/Max & $44 / 180$ & $35 / 180$ & \\
\hline $\begin{array}{l}{ }^{*} \text { Two sample t-te } \\
{ }^{* \star} \text { Non-parametri }\end{array}$ & lilkoxon rank sum & & \\
\hline
\end{tabular}

Reasons for failure

The reasons for failure according to the failure criteria are reported in Table 2 as percentages of the total failures per group.

Table: 2: Failures according to criteria, indicated by numbers and percentages

\section{Reason for failure}

FRCSM debonding at enamel-composite interface/

BLSM debonding at enamel-cement interface

FRCSM debonding at composite-fibre interface/

BLSM debonding at cement-band interface

Fracture of the fibre/ wire loop

FRCSM

n (\%)

BLSM

Bending of the fibre/ wire to impinge on soft tissue

Total failures Loss of contact with adjacent tooth
$2(40 \%)$

$1(20 \%)$

$2(40 \%)$

$1(20 \%)$

$4(80 \%)$

$5(100 \%) \quad 5(100 \%)$
Debonding of the fibre-composite interface accounted for $20 \%$ of the FRCSM device failures reported. Previous authors have also reported debonding of the fibrecomposite interface as a reason for FRCSM failure, attributing respectively $4.2 \%{ }^{1}$ and $13.3 \%{ }^{10}$ of all recorded FRCSM failures to this cause. The debonding might be the result of strain placed on the fibre-composite bond during finishing, occlusal contact with the fibre strut, and wearing of the composite layer by the forces of mastication. . $^{1,10}$ Differences in bonding agents, placement techniques, types of composite and operator skill might all have contributed to the variation in these results, as FRCSM-related techniques are not yet standardized.

Fracturing of the fibre frame accounted for $40 \%$ of the FRCSM failures. Previous studies had attributed $6.7 \%{ }^{10}$ and $16.7 \%{ }^{1}$ of device failures to such fracturing. Fibre frames are thought to fracture through mechanical stresses arising from the chewing of hard/sticky foods, and/ 


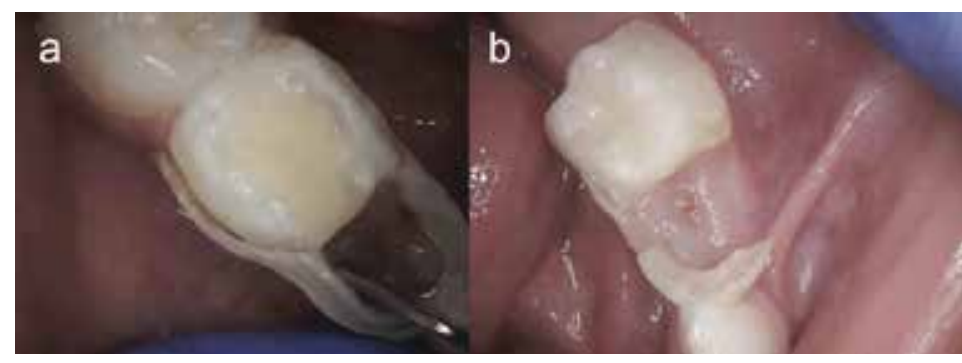

Figure 2: Examples of (a) bond failure on the functional cusp side (the visible metal instrument is being used to demonstrate the loose fibre bundle); (b) fibre fracture on the functional cusp side.

or the over-eruption of the tooth opposing the edentulous area, which subsequently increases and concentrates masticatory forces on the fibre.1,10,16 In the present study, both failed frames broke on the side of the functional cusp, as illustrated in Figure 2b. This finding supports Baroni et al. ${ }^{17}$ conclusions that the mechanical stresses to which the appliance is subjected are more important to its long-term success than is its design. Consequently, the effects of masticatory forces and clearance between the fibre and the opposing tooth, especially when the fibre is bonded to the functional sides, should be taken into consideration during the placement of an FRCSM. Improving the bond strength on the functional side of a deciduous abutment tooth through, for example, adding mechanical retention by embedding the fibre and composite into a prepared groove could be advantageous and merits further exploration.

Interestingly, both FRCSM devices that fractured in this study retained contact with their non-abutment teeth (Figure $2 b)$. Thus, although the devices were reported as failures according to the failure criteria, clinically they still fulfilled their space-maintaining purpose. The unlikely success of these two "broken" fibres could indicate the possibility of placing half a loop, bonded to the non-functional side of the abutment tooth, as the loop seems to fracture on the functional cusp side. Indeed, Kirzioğlu and Ertürk ${ }^{4}$ have previously suggested using a single fibre bonded to the non-functional side of both teeth. Whilst it might prove challenging to place half a loop without bonding it to the anterior tooth, this approach could be investigated further.

Bending of the fibre to impinge on soft tissue was not reported in this study as a reason for any FRCSM failures. In fact, no other clinical FRCSM studies have reported fibre bending as a reason for device failure..$^{1,4,11}$

BLSM failure rates are in accordance with the results of a study ${ }^{10}$ that reported a $63.3 \%$ failure over a six-month period. In previous studies, cement loss was found to be the main reason (i.e. $46.7 \%^{10}$ and $60 \%{ }^{1,5}$ ) for failures of fixed space maintainers. In the present study, only one band was found to be de-cemented. The reasons for these disparate outcomes might include cement type, band fit and moisture control. Croll ${ }^{18}$ suggested using zinc phosphate or polycarboxylate cement to secure bands. However, GIC, which enables bonding to metal and enamel with the additional advantage of fluoride release, is now more widely accepted. ${ }^{5}$ The cement chosen for this study was a GIC that is specifically indicated for the cementation of orthodontic devices. Some of the studies reviewed did not indicate the type of cement used. ${ }^{1}$ Therefore, it is possible that the use of more moisture-sensitive cements, or cements that are not indicated for orthodontic band cementation, could have contributed to the high de-cementation rates reported by other research, ${ }^{1,10}$ although the results may also have been significantly influenced by operator skills and placement techniques.

The main reason for failure (80\%) in this study was bending of the loop to impinge on soft tissue as illustrated in Figure 3. Distortion of the loop has been reported in two previous studies as a failure in, respectively, $3.3 \%{ }^{10}$ and $9 \%{ }^{7}$ of cases. Intermittent functional loading on the space maintainer causes high compressive stresses on the tooth supporting the cantilever extension. ${ }^{19}$ Losing contact with the non-attached abutment tooth has been suggested as the major factor leading to bending of a loop with subsequent submerging of the wire beneath the gingiva. ${ }^{18,20}$ Previous BLSM studies did not limit placement to deciduous molars and included those placed on permanent teeth. Therefore the loop extended further, giving the cantilever wire a larger contact surface in comparison with the situation in this study, where all the wires extended to a deciduous canine with a smaller contact area. The absence of a rest could have contributed to the instability of the loop.

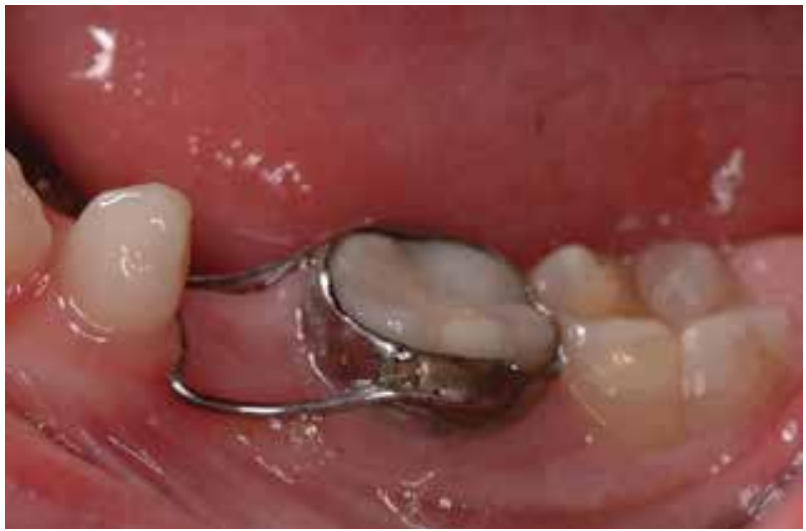

Figure 3: BLSM with bent wire impinging on soft tissue with signs of inflammation.

Second, the thickness of the wire used for construction of BLSMs was not specified in previous studies comparing BLSMs with FRCSMs. ${ }^{1,10}$ For this study a $0.8 \mathrm{~mm}$ diameter stainless steel round wire was used, as described by Kara et al. $^{20}$ The findings from the current study may indicate that this wire thickness is inadequate for BLSMs without an occlusal rest. Although wire thickness is specified for active orthodontic devices, no specification could be found specifically for BLSMs. Further research could recommend a suitable wire thickness specifically for BLSMs with and without occlusal rests.

Sasa et al. $^{7}$ also suggested that children fiddling with devices could be a possible reason for distortion of the wires. During the current study, one child admitted to playing with the wire because it felt 'funny' in his mouth. Previous authors ${ }^{7,10}$ did not record bending of a wire separately as a reason for failure; consequently, it might have been recorded under the formation of soft tissue lesions or as unspecified reasons. These considerations might explain why bending of the wire has not been recorded as a main reason for failure in previous studies. ${ }^{1,7,10}$ 
The present study attributed only one (20\%) failure to a loop fracture. Previous BLSM studies have reported $6.7 \%^{1,10}$ and $9 \%^{7}$ failure rates over six months. Another study with a 40-month follow-up period reported a higher incidence of $22.2 \%,{ }^{2}$ which indicates that the incidence of fracture might increase with time. Fracture of the metal loop is commonly attributed to poor-quality construction. Factors that might have an influence during construction include an incomplete solder joint, overheating of the wire, over-thinning of the joint or thinning of the wire during polishing..$^{5,10,21}$

Reasons for BLSM failure not found in this study but reported elsewhere include slippage of the band gingivally, ${ }^{10}$ split bands and unspecified causes. $^{5}$ It is important to note that all BLSM cases that failed in this study were not reparable chairside.

In addition to failures, this study delivered coincidental clinical findings. It became evident that FRCSMs were more appealing to the parents of the patients. Six of the parents (30\%) immediately commented that, although they would adhere to the allocation process, they preferred an FRCSM for its aesthetics and the need for only one appointment for its placement.

Four participants in this study presented with two missing first deciduous molars. Two received FRCSM and two, BLSM. All four commented that they preferred the FRCSM. Their preference might have been due to the FRCSM's superior aesthetics, the ease of its placement, and/or comfort.10,11,22,23 However, when patients were asked to elaborate, it emerged that the main reason for their preference was the discomfort experienced during BLSM band fitting and impression taking. This finding is in agreement with results reported by Garg et al., ${ }^{10}$ who used the Wong-Baker Face Pain Rating Scale to identify patient preference during a split-mouth study comparing the FRCSM with the BLSM. They confirmed that the FRCSM was the patients' preferred device.

Similar to the experience of other researchers, ${ }^{10}$ impression taking for BLSMs proved challenging with some children. One patient cried during the taking of an impression, while another could not tolerate the impression material in the maxilla because of a gag reflex. In the latter case, it was decided to fit an FRCSM instead.

The greater the time lapse between the extraction and placement of a space maintainer the greater is the incidence of space loss. ${ }^{24}$ Indeed, in this study, it proved

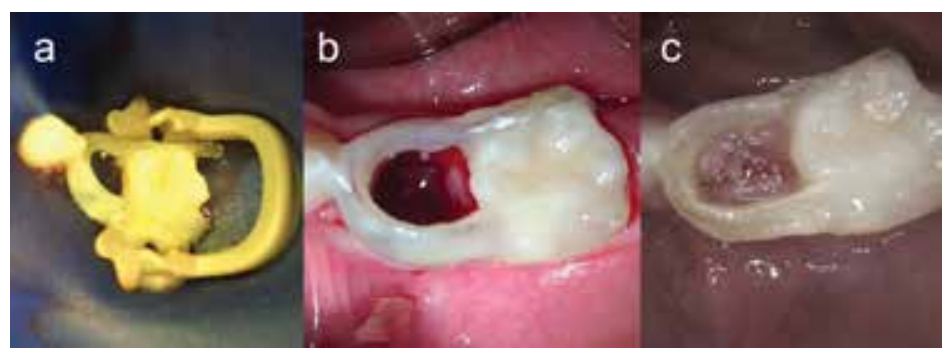

Figure 4: (a) Placement in theatre, rubber dam applied directly after an extraction: (b) Placement in theatre, directly after an extraction; (c) one week follow-up. convenient to place FRCSMs in theatre directly after an extraction. Bleeding was controlled and the rubber dam was positioned, enabling the FRCSM to be placed immediately. Follow-up visits indicated normal healing of the extraction socket. (See Figure 4.)

Placement of FRCSMs proved to be technique sensitive. Manipulation of the fibres to form a uniform loop was a challenge. As a result, not all fibres had a perfect loop shape (as illustrated in Figure 5a). However, this did not prove to affect the FRCSM failure rate in this small sample, as even the non-uniform fibre loop was intact after six months.

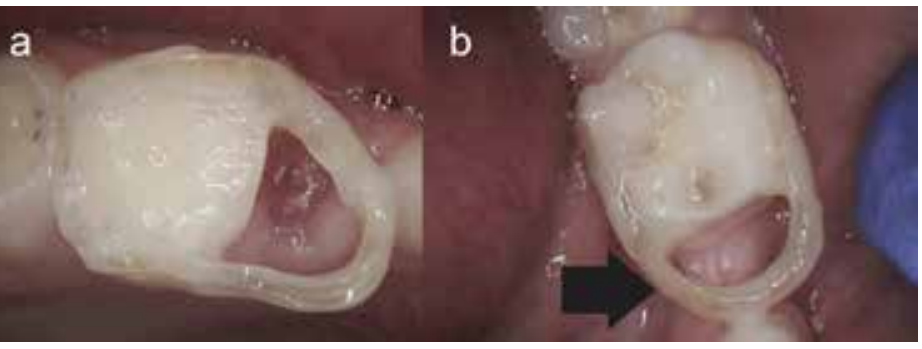

gure 5: (a) Skew orientation of a fibre loop during placement; (b) chipping of composite the fibre frame

During the study, it became evident that the composite covering the fibre would chip off over time (Figure 5b). Although this was not reported as an FRCSM failure in this study, it has previously been reported as a type of device failure. ${ }^{1}$ Chipping of the composite could influence plaque retention, patient comfort, device strength, and device longevity. Repairs can of course be effected chairside with flowable composite.

Considering all the findings from this study, both the BLSM and FRCSM have obvious limitations- and the search for a more economic, aesthetic and effective fixed space maintainer may still be warranted.

\section{CONCLUSIONS}

Based on the results of this study, the following conclusions can be made:

1. No statistically significant differences were found between the failure rates of the BLSM and the loopdesign FRCSM when placed on deciduous molars.

2. A $0.8 \mathrm{~mm}$ diameter stainless steel round wire for construction of the BLSM is not efficient.

3. The effectiveness of the loop-design FRCSM is limited by bond strength and further research on the technique is recommended.

\section{Acknowledgements}

The materials were supplied by Stick Tech Ltd/ GC Corporation, Turku, Finland, and 3M ESPE, Johannesburg, South Africa. No additional source of funding was used.

\section{References}

1. Subramaniam P, Babu GKL, Sunny R. Glass fiber-reinforced composite resin as a space maintainer: a clinical study. J Indian Soc Pedod Prev Dent. 2008; 26:98-103.

2. Kargul B, Çaglar E, Kabalay U. Glass fiber reinforced composite resin space maintainer: case reports. J Dent Child. 2003;70:258-61.

3. Kulkarni G, Lau D, Hafezi S. Development and testing of fiber-reinforced composite space maintainers. J Dent Child. 
2009;76:204-8.

4. Kırzıoğlu Z, Ertürk Ö, Semra M. Success of reinforced fiber material space maintainers. J Dent Child. 2004;71:158-62.

5. Moore TR, Kennedy DB. Bilateral space maintainers: A 7-year retrospective study from private practice. Pediatr Dent. 2006;28:499-505.

6. Qudeimat MA, Fayle SA. The longevity of space maintainers: a retrospective study. Pediatr Dent. 1998;20:267-72.

7. Sasa IS, Hasan AA, Qudeimat MA. Longevity of band and loop space maintainers using glass ionomer cement: a prospective study. Eur Arch Paediatr Dent. 2009;10:6-10.

8. Rudo DN, Karbhari VM. Physical behaviors of fiber reinforcement as applied to tooth stabilization. Dent Clin North Am. 1999;43:7-35.

9. Yeluri R, Munshi AK. Fiber reinforced composite loop space maintainer: An alternative to the conventional band and loop. Contemp Clin Dent. 2012;3:26-8.

10. Garg A, Samadi F, Jaiswal JN, Saha S. 'Metal to resin': A comparative evaluation of conventional band and loop space maintainer with the fiber reinforced composite resin space maintainer in children. J Indian Soc Pedod Prev Dent. 2014;32:111-6.

11. Kargul B, Çaglar E, Kabalay U. Glass fiber-reinforced composite resin as fixed space maintainers in children: 12-month clinical follow-up. J Dent Child. 2005; 72:109-12.

12. Zilberman $U$, Lasilla $L$. The use of glass-fibers, ribbon and composite for prosthetic restoration of missing primary teeth - laboratory and clinical research. Open Dent J. 2014;8:220-8.

13. Sticktech Ltd. [Internet]. Stick Tech - brochures. [Cited 2015 Oct]. Available from: http://www.sticktech.com/contentlibrary/ pdfs/brochure_pdfs/5\%201010\%20CB\%20Clinical\%20 Guide\%20updated\%202011-02\%20low\%20res.pdf.

14. Peutzfeldt A, Nielsen LA. Bond strength of a sealant to primary and permanent enamel: phosphoric acid versus self-etching adhesive. Pediatr Dent. 2004;26: 240-4.

15. Artun J, Marstrander PB. Clinical efficiency of two different types of direct bonded space maintainers. ASDC J Dent Child. 1983;50:197-204.

16. Ngan P, Alkire RG, Fields H. Management of space problems in the primary and mixed dentitions. J Am Dent Assoc. 1999;130:1330-9.

17. Baroni C, Franchini A, Rimondini L. Survival of different types of space maintainers. Pediatr Dent. 1994;16:360-1.

18. Croll TP. Prevention of gingival submergence of fixed unilateral space maintainers. ASDC J Dent Child. 1982;49:48-51.

19. White SN, Caputo AA, Anderkvist T. Effect of cantilever length on stress transfer by implant-supported prostheses. J Prosthet Dent. 1994;71:493-9.

20. Kara NB, Çehreli S, Sağırkaya E, Karasoy D. Load distribution in fixed space maintainers: a strain-gauge analysis. Pediatr Dent. 2013;35:19E-22E.

21. Rajah LD. Clinical performance and survival of space maintainers: Evaluation over a period of 5 years. J Dent Child. 2002; 69:156-60

22. Karaman Al, Kir N, Belli S. Four applications of reinforced polyethylene fiber material in orthodontic practice. Am J Orthod Dentofacial Orthop. 2002;121: 650-4.

23. Tuloglu N, Bayrak S, Tunc ES. Different clinical applications of bondable reinforcement ribbon in pediatric dentistry. Eur $\mathrm{J}$ Dent. 2009;3:329.

24. Owen DG. The incidence and nature of space closure following the premature extraction of deciduous teeth: a literature survey. Am J Orthod. 1971;59:37-49. 